\title{
A new grid of NLTE accretion-disc models for AM CVn systems: application to CE 315
}

\author{
T. Nagel, T. Rauch, and K. Werner \\ Institut für Astronomie und Astrophysik, Kepler Center for Astro and Particle Physics, Eberhard-Karls-Universität, Sand 1, \\ 72076 Tübingen, Germany \\ e-mail: nagel@astro.uni-tuebingen.de
}

Received 21 December 2008 / Accepted 12 March 2009

\begin{abstract}
Context. AMCVn stars are very compact interacting binary systems with helium-dominated spectra. The nature of the donor star remains unclear, but the accretion disc represents the chemical composition of the donor's atmosphere. Analysing the disc will therefore help us to understand the donor star and the formation of these systems.

Aims. We investigate the influence of primary mass, mass-accretion rate, chemical abundances, irradiation by the primary, and inclination on the spectrum of the accretion disc to determine the extent to which it is possible to deduce these system properties by comparison with observed spectra.

Methods. We compute a grid of metal-line blanketed NLTE accretion-disc spectra for an extensive parameter space by solving selfconsistently the radiative-transfer equations and the equations describing the vertical structure using our accretion disc code $A c D c$. Results. We detect emission-line spectra for low and absorption-line spectra for high mass-accretion rates. Irradiation of the accretion disc by the primary has almost no influence on the spectrum. The spectroscopic detection of the primary is achievable most easily in the UV. Comparing an observed spectrum of CE 315 with our accretion-disc models, we find the qualitatively closest match for a $0.8 M_{\odot}$ primary and a mass-accretion rate of $10^{-11} M_{\odot} / \mathrm{yr}$. Furthermore, the disc of CE 315 exhibits a strong silicon underabundance confirming the hypothesis that it is a Pop. II object.
\end{abstract}

Key words. accretion, accretion disks - navae, cataclysmic variables - stars: individual: CE 315

\section{Introduction}

AMCVn stars are close, interacting binary systems with very short orbital periods below the $78 \mathrm{~min}$ period minimum of classical, hydrogen cataclysmic variables (CVs). Their spectra are dominated by helium lines. They are named after the prototype AM CVn (also known as HZ 29), whose binarity was proposed for the first time by Smak (1975). Presently, 21 of these systems are known (Table 1), seven of them having been discovered by the Sloan Digital Sky Survey (Anderson et al. 2005; Roelofs et al. 2005). The accretor is a white dwarf (WD), whereas the nature of the Roche-lobe-filling low-mass $\left(M_{2}<0.1 M_{\odot}\right)$ donor remains unclear. It might be a helium white dwarf, a helium star, or the helium-rich core of an evolved CV secondary (for more details see, e.g., Nelemans 2005). Since the matter in the accretion disc originates in the outer layers of the donor star, the analysis of the disc and the determination of its chemical composition will help us to understand the nature of the donor and the formation channel of AMCVn systems.

AM CVn systems are discussed to contribute significantly to the Type Ia supernova production rate (Nelemans et al. 2001; Solheim \& Yungelson 2005). Furthermore, they are sources of low-frequency gravitational wave radiation, which will be detectable with the future Laser Interferometer Space Antenna LISA (e.g., Hils \& Bender 2000).

The AMCVn stars can be divided into subgroups according to their orbital periods. The first group includes systems with ultra-short periods below $10 \mathrm{~min}$. They are probably direct-impact accretors without an accretion disc. The shortperiod group $\left(P_{\text {orb }}<20 \mathrm{~min}\right)$ with high mass-accretion rates of about $10^{-8} M_{\odot} / \mathrm{yr}$ exhibits mostly absorption-line spectra, whereas the long-period group $\left(P_{\text {orb }}>35 \mathrm{~min}\right)$ with low massaccretion rates of about $10^{-11} M_{\odot} /$ yr exhibits emission-line spectra. In-between are the outbursting systems, which undergo dwarf-nova cycles like hydrogen CVs but on shorter timescales because of their much smaller accretion discs. Depending on their state in the dwarf-nova cycle, they show emission- or absorption-line spectra.

There have been various attempts to model the accretion disc in AMCVn systems. Tsugawa \& Osaki (1997) calculated the thermal-tidal instability cycle for helium discs. Kunze et al. (2001) studied the interaction between the infalling gas stream and the disc. Semionovas \& Solheim (1999) calculated a model grid of hydrogen-helium NLTE discs and applied it to four of the AM CVn systems, El-Khoury \& Wickramasinghe (2000) calculated a LTE model grid of hydrogen-helium discs in the optical range, and applied it to AM CVn and CR Boo. Nasser et al. (2001) calculated NLTE H/He-disc spectra for some AM CVn systems in the optical wavelength range, varying the massaccretion rate only slightly $\left(2-9 \times 10^{-9} M_{\odot} / \mathrm{yr}\right)$. Nagel et al. (2004) analysed the optical spectrum of AMCVn itself, using their own NLTE disc models containing $\mathrm{H}, \mathrm{He}, \mathrm{C}, \mathrm{N}, \mathrm{O}$, and $\mathrm{Si}$.

In this work, we calculated an extended grid of NLTE accretion-disc models, containing $\mathrm{H}, \mathrm{He}, \mathrm{C}, \mathrm{N}, \mathrm{O}$, and $\mathrm{Si}$, to study the influence of parameters such as primary mass, massaccretion rate, or chemical abundances on the disc spectrum. The 
Table 1. The known AMCVn systems.

\begin{tabular}{lcl}
\hline \hline System & $P_{\text {orb }}(\mathrm{s})$ & Type \\
\hline RX J0806.3+1527 & $321.52911(2)$ & ultra-short, pulsed X-ray \\
V407 Vul & 569.4 & ultra-short, pulsed X-ray \\
\hline ES Cet & 621 & high-state \\
AM CVn & 1028.73 & high-state \\
HP Lib & 1103 & high-state \\
\hline CR Boo & 1471 & outbursting \\
KL Dra & 1500 & outbursting \\
V803 Cen & 1596 & outbursting \\
SDSS J0926+3642 & 1699 & outbursting \\
CP Eri & 1701 & outbursting \\
2003aw (V406 Hya) & 2028 & outbursting \\
2QZ J1427-01 & $2194(?)$ & outbursting \\
\hline SDSS J1240-0159 & 2242 & low-state \\
GP Com & 2794 & low-state \\
CE 315 (V396 Hya) & 3906 & low-state \\
\hline SDSS J1411+4812 & 2760 & emission lines \\
SDSS J1552+3201 & $3376.3(3)$ & emission lines \\
SDSS J0129+3842 & - & emission lines \\
SDSS J1208+3550 & - & emission lines \\
SDSS J2047+0008 & - & absorption lines \\
SNF 20060524-042 & - & - \\
\hline
\end{tabular}

Data taken from Bildsten et al. (2006) and references therein, Roelofs et al. (2007a,b), Anderson et al. (2005, 2008), and the website of G. Nelemans (http://www . astro.ru.nl/ nelemans/).

values of primary mass and mass-accretion rate were selected to include the variety of AMCVn systems. It is the first time that a such an extensive helium-dominated model grid, including the effects fo metals, has been calculated for wavelengths between the EUV and IR.

In the next section, we briefly introduce our modelling of accretion discs and their spectra. In Sect. 3, we present vertical structures and spectra of the accretion discs, varying the primary mass, mass-accretion rate, chemical abundances, and inclination angle. We also consider the effect of irradiation of the disc by the primary for different cases and the possibility of a spectroscopic detection of the primary. Finally, we compare our model spectra with an observed spectrum of CE 315 (Sect. 4). We close with a brief summary in Sect. 5 .

\section{Accretion-disc modelling}

For the calculation of the metal-line blanketed NLTE accretiondisc models, we use our accretion-disc code $A c D c$ (Nagel et al. 2004). It is based on the radial structure of an $\alpha$-disc (Shakura \& Sunyaev 1973), assuming a stationary, geometrically thin disc (total disc thickness $H$ is much smaller than the disc diameter). This allows the decoupling of the vertical and radial structures and, together with the assumption of axial symmetry, we can separate the disc into concentric annuli of plane-parallel geometry. In that way, the radiative transfer becomes a one-dimensional problem. The mass of the disc is far smaller than the mass of the central object, so we can neglect self-gravitation. The radial distribution of the effective temperature $T_{\text {eff }}$ can be described by

$T_{\mathrm{eff}}(R)=\left[\frac{3 G M_{1} \dot{M}}{8 \pi \sigma R^{3}}\left(1-\sqrt{\frac{R_{1}}{R}}\right)\right]^{1 / 4}$,

where $M_{1}$ and $R_{1}$ denote the mass and the radius of the central object, $\dot{M}$ is the mass-accretion rate, $G$ the gravitational constant, and $\sigma$ the Stefan-Boltzmann constant. The radial profile of surface mass density $\Sigma$ can be described by

$w \Sigma(R)=\frac{\dot{M}}{3 \pi}\left(1-\left(\frac{R_{1}}{R}\right)^{1 / 2}\right)$

for the kinematic viscosity $w$.

For each disc ring, the following set of coupled equations were solved simultaneously under the constraints of particle number and charge conservation:

- radiation transfer for the specific intensity $I$

$\mu \frac{\partial I(v, \mu, z)}{\partial z}=-\chi(v, z) I(v, \mu, z)+\eta(v, z)$

with the absorption coefficient $\chi$, the emission coefficient $\eta$, the geometrical disc height $z$ above the midplane, and $\mu=\cos \theta$ with $\theta$ being the angle between the ray and $z$. To consider irradiation of the accretion disc by the primary, the irradiation angle and the spectrum of the primary (blackbody or detailed model-atmosphere spectrum) must be specified. Since we employ a five-point Gaussian quadrature for angle integration, the stellar disc is usually unresolved (except for annuli very close to the star) and the irradiated flux is assigned to a single quadrature point. Stellar limb darkening is neglected;

- hydrostatic equilibrium between gravitation, gas pressure $P_{\text {gas }}$, and radiation pressure

$\frac{\mathrm{d} P_{\text {gas }}}{\mathrm{d} m}=\frac{G M_{1}}{R^{3}} z-\frac{4 \pi}{c} \int_{0}^{\infty} \frac{\chi(v)}{\rho} H(v, z) \mathrm{d} v$,

with $\rho$ denoting the mass density, and $H$ the Eddington flux. Here we also introduced the column mass depth $m$ as

$m(z)=\int_{z}^{\infty} \rho\left(z^{\prime}\right) \mathrm{d} z^{\prime} ;$

- energy balance between the viscously generated energy $E_{\text {mech }}$ and the radiative energy loss $E_{\text {rad }}$

$E_{\text {mech }}=E_{\text {rad }}\left(+E_{\text {conv }}\right)$

with

$E_{\text {mech }}=w \Sigma\left(R \frac{\mathrm{d} \omega}{\mathrm{d} R}\right)^{2}=\frac{9}{4} w \Sigma \frac{G M_{1}}{R^{3}}$

and

$E_{\mathrm{rad}}=4 \pi \int_{0}^{\infty}(\eta(v, z)-\chi(v, z) J(v, z)) \mathrm{d} v$

with the angular velocity $\omega$, and the mean intensity $J$. Convection $\left(E_{\text {conv }}\right)$ is neglected in our models;

- NLTE rate equations for the population numbers $n_{i}$ of the atomic levels $i$

$\frac{\partial n_{i}}{\partial t}=n_{i} \sum_{i \neq j} P_{i j}-\sum_{j \neq i} n_{j} P_{j i}$.

$P_{i j}$ denotes the rate coefficients, consisting of radiative and collisional components. For the static case, it follows that

$\frac{\partial n_{i}}{\partial t}=0$ 
Table 2. Statistics of the model atoms used in our disc models.

\begin{tabular}{lrrr}
\hline \hline Ion & LTE levels & NLTE levels & Lines \\
\hline H I & 6 & 10 & 45 \\
H II & - & 1 & - \\
He I & 15 & 29 & 61 \\
He II & 24 & 8 & 28 \\
He III & - & 1 & - \\
C I & 14 & 7 & 4 \\
C II & 4 & 38 & 160 \\
C III & 9 & 58 & 329 \\
C IV & 49 & 9 & 17 \\
C V & 0 & 1 & 0 \\
N I & 15 & 12 & 15 \\
N II & 4 & 17 & 21 \\
N III & 38 & 7 & 5 \\
N IV & 78 & 16 & 30 \\
N V & 8 & 54 & 297 \\
N VI & 0 & 1 & 0 \\
O I & 12 & 1 & 0 \\
O II & 14 & 29 & 82 \\
O III & 8 & 36 & 42 \\
O IV & 58 & 11 & 5 \\
O V & 15 & 6 & 4 \\
O VI & 0 & 1 & 0 \\
Si II & 5 & 25 & 28 \\
Si III & 17 & 17 & 27 \\
Si IV & 11 & 28 & 49 \\
Si V & 44 & 15 & 20 \\
Si VI & 0 & 1 & 0 \\
\hline & & &
\end{tabular}

By integrating the spectra of the individual annuli, one obtains a complete disc spectrum for different inclination angles, where the spectral lines are Doppler shifted according to the radial component of the Kepler rotational velocity. For a more detailed description of the accretion-disc modelling with $A c D c$, we refer the reader to Nagel et al. (2004).

The input parameters we have to provide for the calculation of disc rings are the mass $M_{1}$ and radius $R_{1}$ of the primary, the mass-accretion rate $\dot{M}$, the Reynolds number Re, the radius of the annulus $R$, the chemical abundances, the irradiation angle $\beta$, and the spectrum or the black-body temperature $T_{\text {irr }}$ of the primary. Detailed information about the involved atomic data is provided in the form of a model atom (cf. Rauch \& Deetjen 2003).

Since the accretion discs of AMCVn stars are fed by a helium-rich secondary, we assume a disc composition dominated by helium. Hydrogen, carbon, nitrogen, oxygen, and silicon are considered with varying abundances (Table 4). Iron-group elements and molecule formation are not considered. Details about our model atoms are shown in Table 2, the silicon lines in the optical and UV are split into multiplets (assuming relative LTE populations for the sublevels).

The model grid presented here comprises more than 70 accretion discs with about 10 single disc-ring models each. For each disc, the spectrum for five inclination angles is calculated.

\section{The model grid}

We calculated a grid of accretion-disc models to cover the entire parameter range of AMCVn systems. We assumed five different primaries from 0.6 to $1.4 M_{\odot}$, for each four different massaccretion rates from $10^{-8}$ to $10^{-11} M_{\odot} / y r$ (see Table 3 ). Every disc spectrum is calculated for at least three different chemical
Table 3. Parameters of the model grid. $M_{1}, R_{1}$ and $g$ are the mass, the radius and the surface gravity of the white dwarf primary, respectively, $\dot{M}$ the mass-accretion rate, $\log g$ is calculated from $M_{1}$ and $R_{1}$.

\begin{tabular}{ccccc}
\hline \hline$\#$ & $M_{1}\left[M_{\odot}\right]$ & $R_{1}[\mathrm{~km}]$ & $\log g$ & $\log \dot{M}\left[M_{\odot} / \mathrm{yr}\right]$ \\
\hline 1 & 0.6 & 9550 & 7.9 & $-8,-9,-10,-11$ \\
2 & 0.8 & 7380 & 8.3 & $-8,-9,-10,-11$ \\
3 & 1.0 & 5830 & 8.6 & $-8,-9,-10,-11$ \\
4 & 1.2 & 4100 & 9.0 & $-8,-9,-10,-11$ \\
5 & 1.4 & 3160 & 9.3 & $-8,-9,-10,-11$ \\
\hline
\end{tabular}

Table 4. Variation in the abundances for the included elements with $[x]=\log ($ mass fraction/solar mass fraction) of species $x$, following Grevesse \& Sauval (2000) for the solar abundances.

\begin{tabular}{cccccc}
\hline \hline$\#$ & {$[\mathrm{C}]$} & {$[\mathrm{N}]$} & {$[\mathrm{O}]$} & {$[\mathrm{Si}]$} & {$[\mathrm{H}]$} \\
\hline 1 & 0 & 0 & 0 & 0 & -10 \\
2 & -3 & 1 & -3 & 0 & -10 \\
3 & -3 & 1 & -3 & -3 & -10 \\
4 & 0 & 0 & 0 & 0 & $-6,-5,-3,-2$ \\
\hline
\end{tabular}

compositions. Unless otherwise noted, all plots shown are of composition \#2 (Table 4).

The maximum outer radius of the discs is defined by the tidal radius, where the disc is truncated by the tidal forces of the secondary. It can approximately be written (Warner 1995) as

$r_{\text {tidal }}=0.60 \cdot \frac{a}{1+q} \quad$ with $\quad 0.03<q<1$

with the binary's separation $a$ and the mass ratio $q=M_{2} / M_{1}$. The separation can be derived from Kepler's law. For the highest mass-accretion rate systems, we assumed an orbital period of $1000 \mathrm{~s}$, for the lowest mass-accretion rate systems $3000 \mathrm{~s}$ and for the systems in between $1500 \mathrm{~s}$. For the companion, we assumed a mass of $0.05 M_{\odot}$ for all systems. In the case of the lowest massaccretion rate, we encountered numerical problems for the outermost disc rings. As a result, the respective disc models were not extended to the tidal radius. Depending on the primary mass and mass-accretion rate, this produced outer disc radii between 8 and $20 R_{1}$.

Figure 1 shows the radial variation in the effective temperature as well as the radial extension for the five primary masses and all mass-accretion rates. Here and in the following, the effective temperatures of the disc are always intrinsic, regardless of whether irradiation is considered or not. Our hottest model has effective temperatures of between $120000 \mathrm{~K}$ and $22000 \mathrm{~K}$, whereas the coolest disc only varies between $9000 \mathrm{~K}$ and $4000 \mathrm{~K}$. As an example, Fig. 2 shows the vertical ionisation structure of an inner disc ring for low and high mass-accretion rates.

\subsection{Variation of the primary mass}

With increasing primary mass and constant mass-accretion rate, the accretion disc becomes hotter, due to the stronger gravitational field of the more compact primary (Fig. 1). In Fig. 3, the vertical structures of a ring in the inner part $\left(1.9 R_{1}\right)$ of the disc are shown for all five primary masses at low mass-accretion rate. In the case of low-mass primaries, the temperature structure becomes nearly isothermal and the disc is optically thin.

Figure 4 shows the optical and UV spectra of the accretion discs for the five primary masses and mass-accretion rates of 


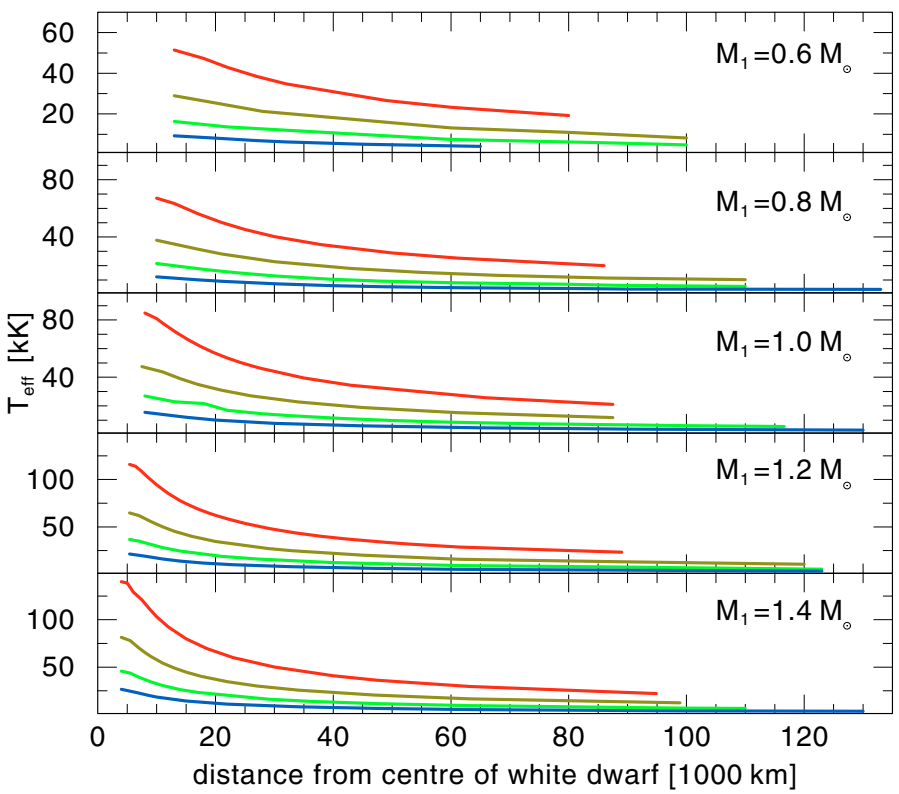

Fig. 1. Radial run of disc effective temperature for five primary masses and four mass-accretion rates (in each panel $\dot{M}=10^{-8}-10^{-11} M_{\odot} / \mathrm{yr}$ from top).

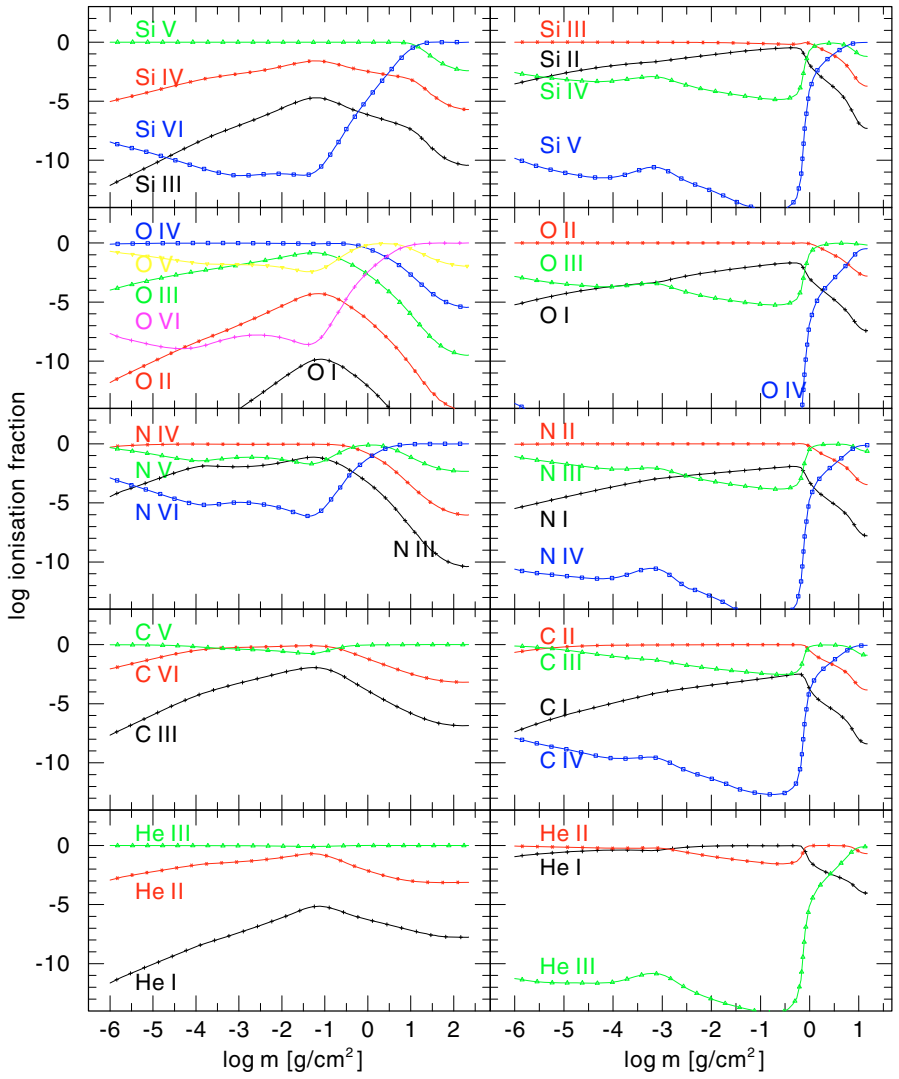

Fig. 2. Vertical run of ionisation fractions for a primary with $0.6 M_{\odot}$ and mass-accretion rates of $10^{-8} M_{\odot} / \mathrm{yr}$ (left panels) and $10^{-10} M_{\odot} / \mathrm{yr}($ right $)$ versus the column mass density (increasing from outer to inner layers) for an inner disc ring $\left(1.4 R_{1}\right)$.

$10^{-8} M_{\odot} /$ yr and $10^{-11} M_{\odot} /$ yr, all shown for the same inclination angle $\left(18^{\circ}\right)$. As expected from observations, the low massaccretion rate models show strong emission-line spectra and the high mass-accretion rate models absorption-line spectra. In the case of low mass-accretion rates, the total flux in the optical and

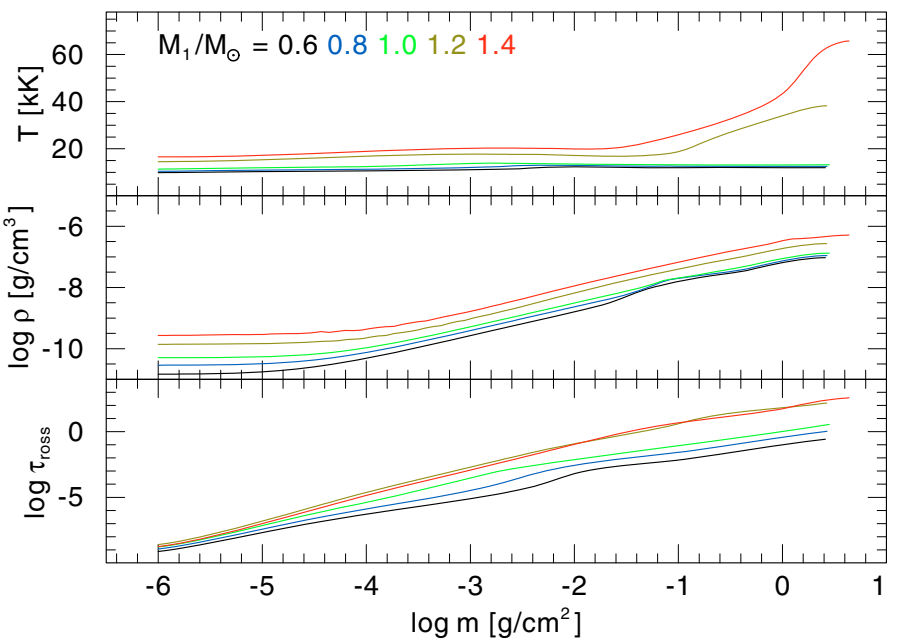

Fig. 3. Vertical structure of an inner disc ring at a radius of $1.9 R_{1}$ for different primary masses at a mass-accretion rate of $10^{-11} M_{\odot} / \mathrm{yr}$. Shown are the temperature $T$ (top panel), the density $\rho$ (middle) and the Rosseland optical depth $\tau_{\text {ross }}$ (bottom).

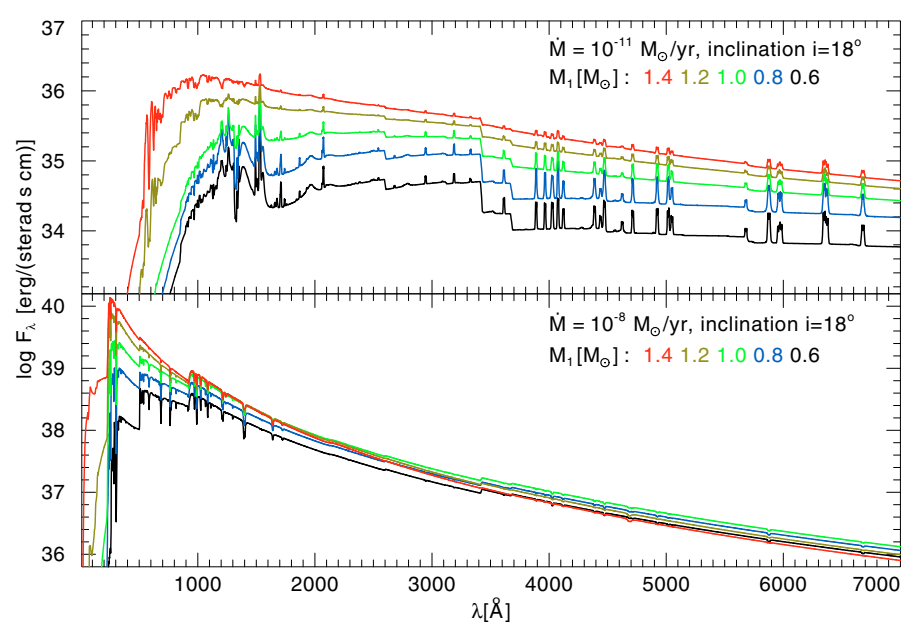

Fig. 4. Accretion-disc spectra for a mass-accretion rate of $10^{-8}$ (bottom panel) and $10^{-11} M_{\odot} / \mathrm{yr}$ (top panel) and different primary masses.

UV increases with increasing primary mass due to the higher disc temperature. In the case of high mass-accretion, the disc model with the most massive primary dominates the UV, but in the optical spectral range the disc fluxes with the lower-mass primaries are higher.

In Fig. 5, the spectra of single rings of a disc with a $0.6 M_{\odot}$ primary and a mass-accretion rate of $10^{-10} M_{\odot} / \mathrm{yr}$ are shown. Due to the different effective temperatures in the inner and outer parts of the disc, the spectra differ significantly and show absorption lines in the inner and emission lines in the outer disc region.

\subsection{Variation of the mass-accretion rate}

An increase in the mass-accretion rate at the same primary mass leads to a higher disc temperature, which can be seen in Fig. 6, where the vertical structures of an inner disc ring $\left(1.9 R_{1}\right)$ are shown for a low-mass primary at four different mass-accretion rates. The surface density increases with increasing accretion rate, as can be seen from the $\log m$ value at the inner depth point. 


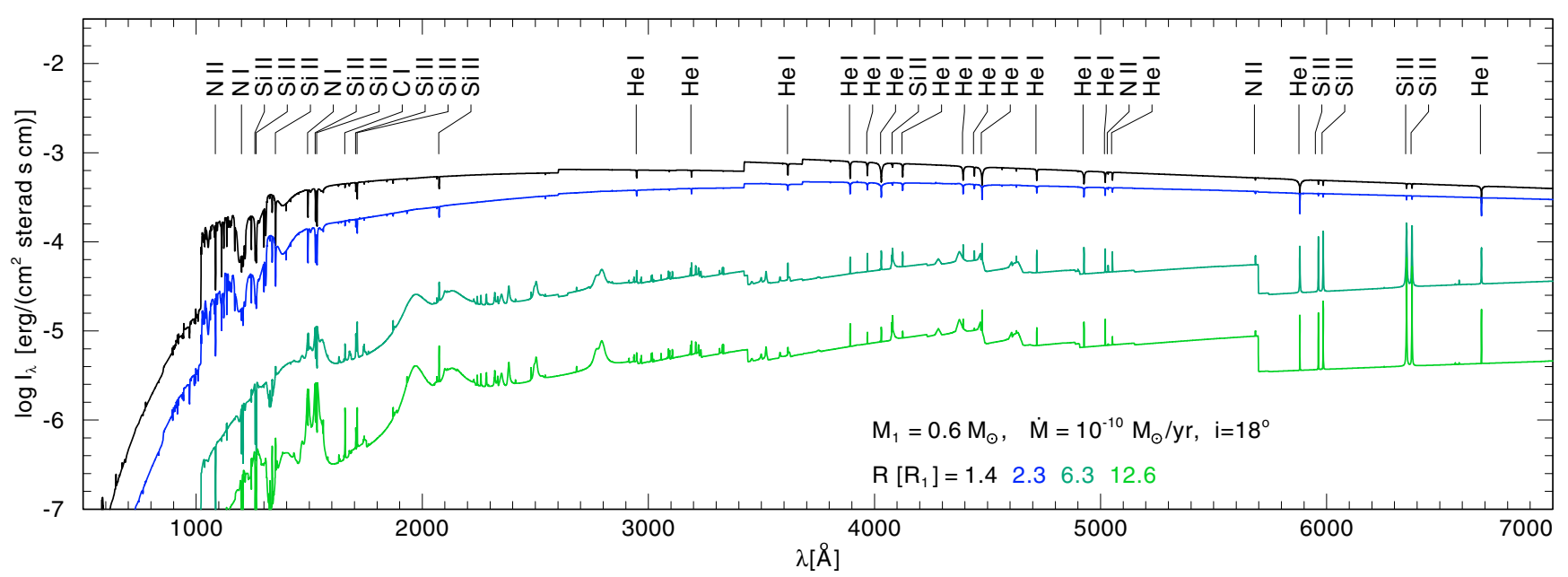

Fig. 5. Disc ring spectra from the inner (top graph, $1.4 R_{1}$ ) to the outer (bottom graph, $12.6 R_{1}$ ) part of the accretion disc for an $0.6 M_{\odot}$ primary and a mass-accretion rate of $10^{-10} M_{\odot} / \mathrm{yr}$ without rotational broadening.

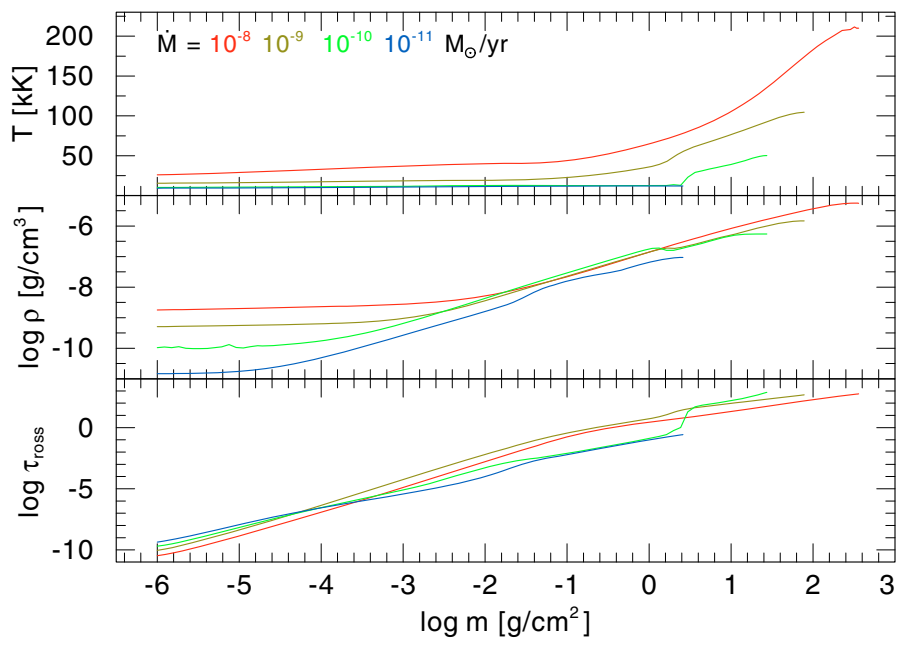

Fig. 6. Vertical structure of an inner disc ring $\left(1.9 R_{1}\right)$ for different massaccretion rates with a WD primary of $0.6 M_{\odot}$.

Figure 7 (lower panel) shows the change from an absorptionline spectrum to an emission-line spectrum with decreasing mass-accretion rate for a low-mass primary of $0.6 M_{\odot}$. Furthermore, the continuum flux level is reduced by almost a factor of 1000 due to the decrease in temperature. In the case of the most massive primary with $1.4 M_{\odot}$, we found a weak emission-line spectrum only for our lowest mass-accretion rate (Fig. 7, upper panel).

\subsection{Variation in chemical abundances}

The variation in the chemical abundances for a low-mass and a high-mass primary is shown in Figs. 8 and 9. We compare models with chemical compositions as listed in Table 4.

In the case of the low-mass primary, there are strong Si lines in the optical, which disappear only for strong Si underabundance. In the case of the high-mass primary, it is difficult to see any differences in the optical disc spectra, but in the UV the disappearance of carbon and silicon lines with corresponding underabundance can be clearly seen. By comparing our model spectra with observations, it should be possible to reveal a metal

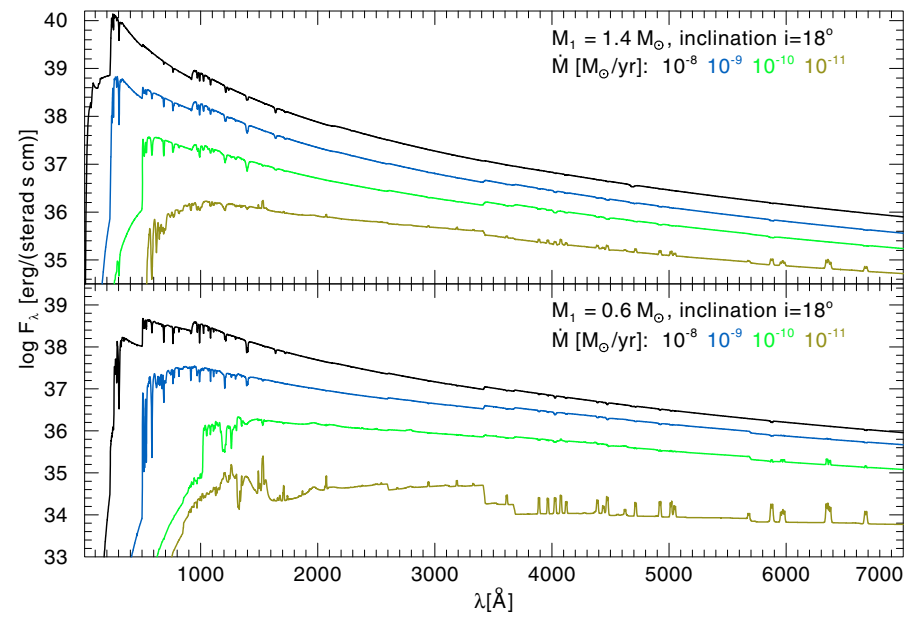

Fig. 7. Accretion-disc spectra for a primary with 0.6 (bottom panel) and $1.4 M_{\odot}(t o p)$ and different mass-accretion rates.

underabundance in the accretion discs of AMCVn systems, as already proposed for GPCom (Marsh et al. 1991; MoralesRueda et al. 2003). The determination of hydrogen in the accretion disc is especially interesting in the case of the whitedwarf formation channel of AMCVn stars, where it is possible that a small amount of hydrogen remains (Podsiadlowski et al. 2003). We increased the abundance of hydrogen in a high and low mass-accretion rate disc for two primaries $\left(0.6\right.$ and $\left.1.2 M_{\odot}\right)$ to deduce the upper limits at which hydrogen becomes visible in the spectrum. We found that in low-state systems a $\mathrm{H}$ abundance of $10^{-6}$ already leads to strong Balmer emission lines (Fig. 9, right panel), whereas in the UV no hydrogen lines are detectable. In high-state systems, only an abundance of $10^{-3}$ produces significant Balmer lines (Fig. 9, left panel). In the UV, even an abundance of $10^{-2}$ is needed to see significant hydrogen lines.

\subsection{Variation of inclination}

All models so far have been shown for an inclination angle of $18^{\circ}$. The variation in the inclination also leads to strong 


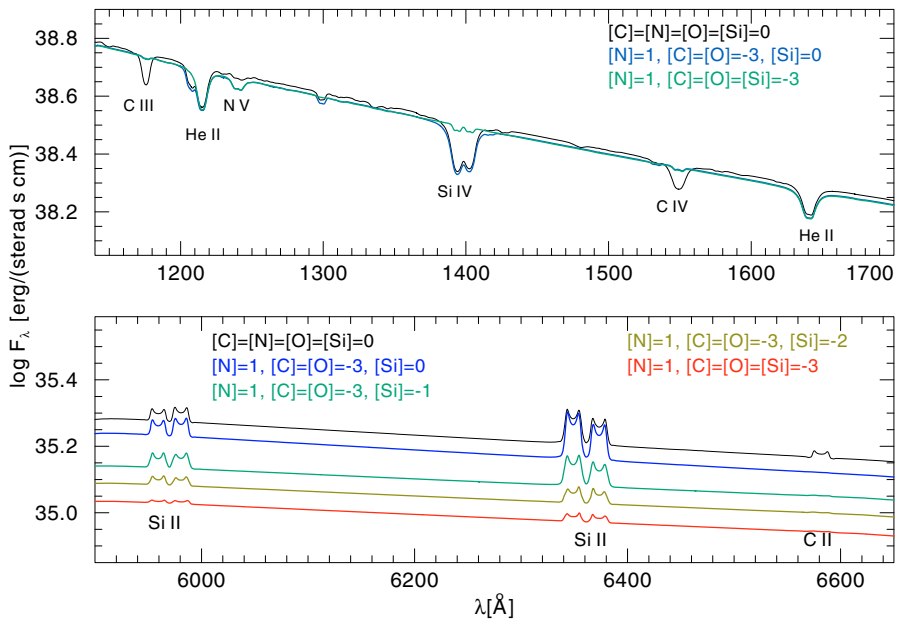

Fig. 8. Accretion-disc spectra for a primary with $1.2 M_{\odot}$ and a massaccretion rate of $10^{-8} M_{\odot} / \mathrm{yr}$ (upper panel) and a primary with $0.6 M_{\odot}$ and a mass-accretion rate of $10^{-10} M_{\odot} / \mathrm{yr}$ (lower) for different chemical abundances.
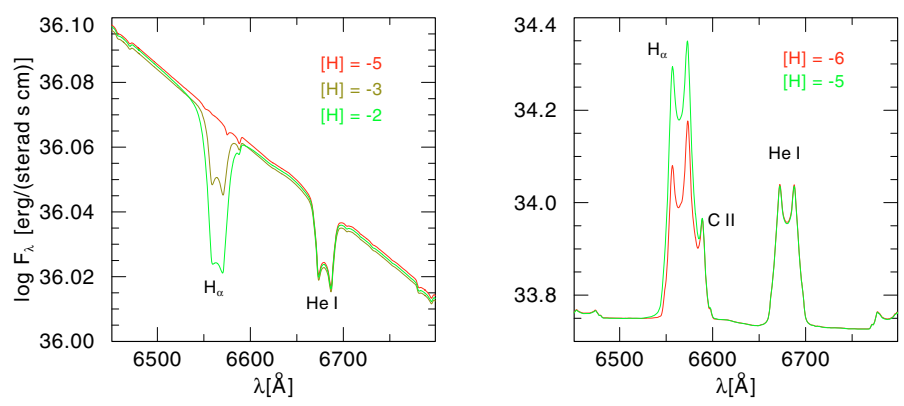

Fig. 9. Accretion-disc spectra for different hydrogen abundances. Left panel: $M_{1}=0.6 M_{\odot}, \dot{M}=10^{-8} M_{\odot} / \mathrm{yr}$, right: $M_{1}=0.6 M_{\odot}, \dot{M}=$ $10^{-11} M_{\odot} / \mathrm{yr}$.

differences in the spectra, which is shown in Fig. 10 for two primary masses. The spectral lines become broader with increasing inclination due to the increasing radial component of the Kepler rotation velocity. At the same time, the total flux decreases because of the smaller visible projected surface area of the disc and limb-darkening effects.

\subsection{Irradiation by the primary}

The geometry of AMCVn systems is quite extreme, as can be seen from Fig. 11, where a sketch of a primary with $0.6 M_{\odot}$ and the accretion disc is shown. This leads to rather large irradiation angles of more than $20^{\circ}$ for the inner part and about $5^{\circ}$ for the outer part of the accretion disc. We accounted for irradiation of the disc by the primary for two primary masses at high and low mass-accretion rate to investigate the influence on the vertical structure and disc spectrum. The irradiation was performed with a $20000 \mathrm{~K}$ black-body spectrum for the low and $50000 \mathrm{~K}$, $80000 \mathrm{~K}$, and $100000 \mathrm{~K}$ for the high mass-accretion rate system. These effective temperatures for the primary are reasonable, following Bildsten et al. (2006), who calculated the heating and cooling of the accreting white dwarf in AM CVn systems. Using white-dwarf model spectra instead of black-body spectra
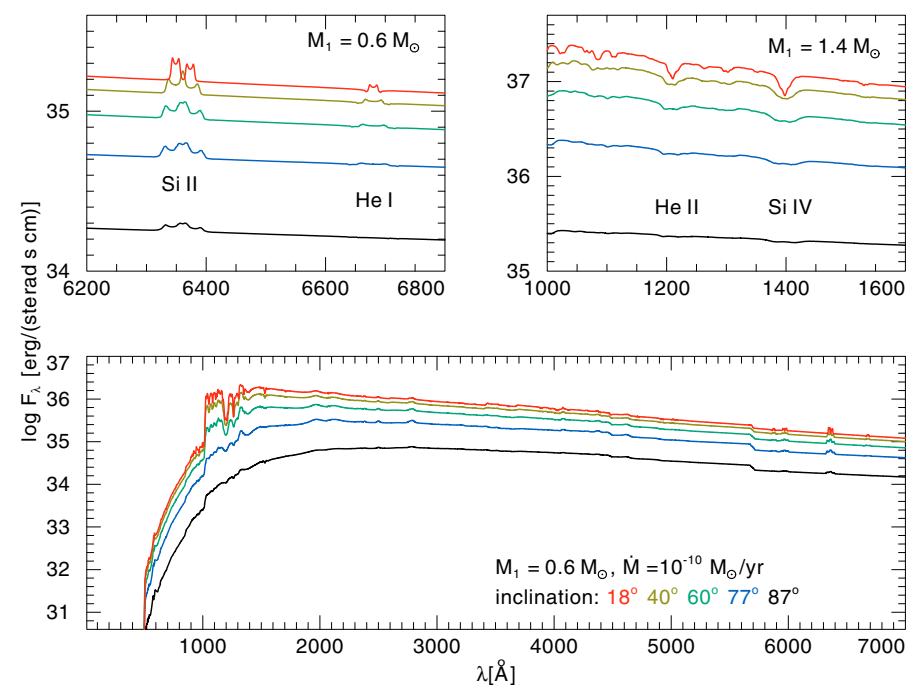

Fig. 10. Accretion-disc spectra for a primary with $0.6 M_{\odot}$ (bottom panel and top left panel) and $1.4 M_{\odot}$ (top right panel) and a mass-accretion rate of $10^{-10} M_{\odot} / \mathrm{yr}$ for different inclination angles.

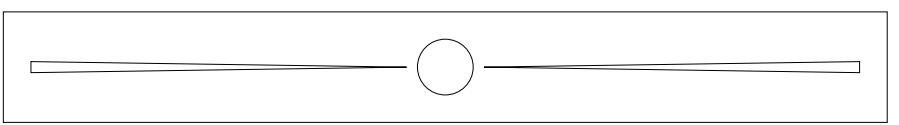

Fig. 11. Sketch of a cut through an AMCVn system (primary with $0.6 M_{\odot}, \dot{M}=10^{-11} M_{\odot} / \mathrm{yr}$, adequate proportions).

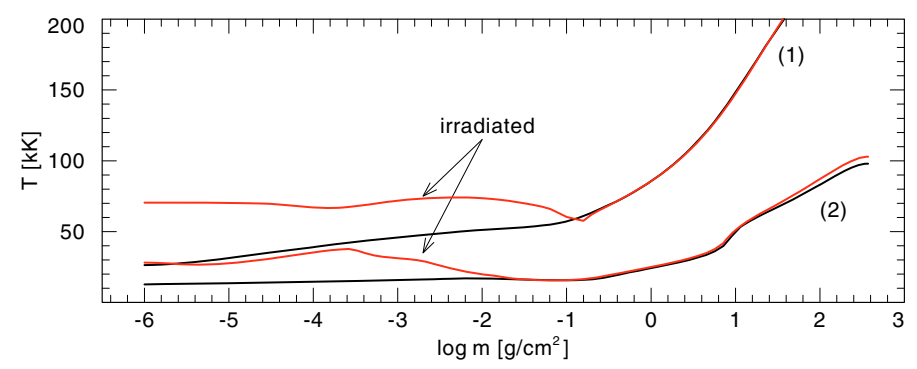

Fig. 12. Temperature structure in the inner $\left(1,1.3 R_{1}\right)$ and outer part $\left(2,12 R_{1}\right)$ of the disc, irradiated by a white dwarf with $T_{\text {eff }}=80000 \mathrm{~K}$ and non-irradiated, primary with $0.8 M_{\odot}$ and mass-accretion rate of $10^{-8} M_{\odot} / \mathrm{yr}$.

for the irradiation does not show any difference in the resulting accretion-disc spectrum.

Figure 12 compares, as an example, the vertical temperature structures of irradiated and non-irradiated disc rings for high mass-accretion rate $\left(10^{-8} M_{\odot} / y r\right)$ in the case of a $0.8 M_{\odot}$ primary. The upper layers of the disc rings are heated up by the irradiation, but the spectra do not significantly differ (not shown). At low mass-accretion rate and with irradiation from a $20000 \mathrm{~K}$ primary, the UV spectrum changes slightly, while the optical spectrum is unaffected (see Fig. 13).

\subsection{Disc and primary}

Since AMCVn systems are very compact binaries, it is impossible to resolve their components. The spectra we obtain 


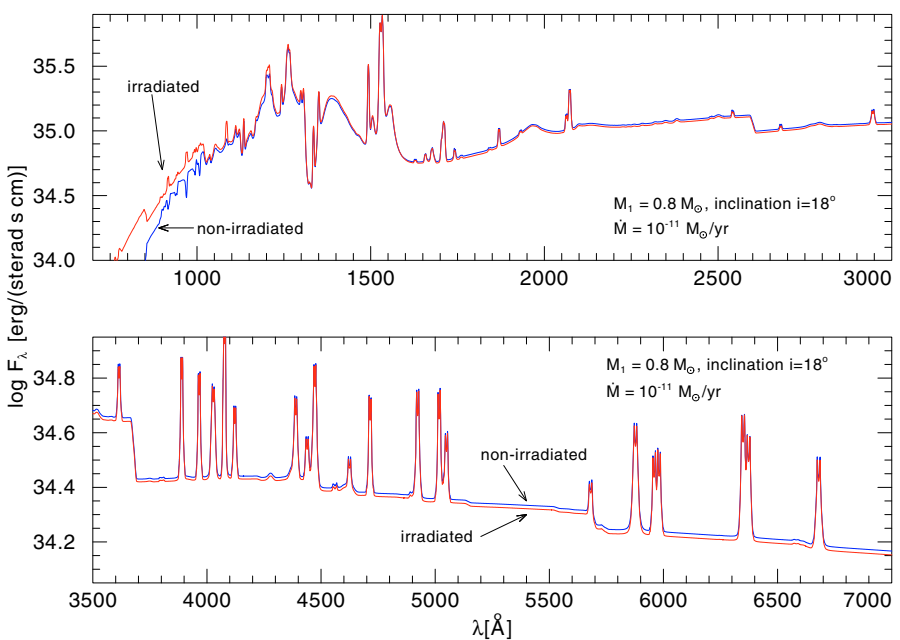

Fig. 13. Comparison of an irradiated $\left(T_{\text {eff, } \mathrm{WD}}=20000 \mathrm{~K}\right)$ and a non-irradiated accretion-disc spectrum for low mass-accretion rate $\left(10^{-11} M_{\odot} / \mathrm{yr}\right)$, primary with $0.8 M_{\odot}$.

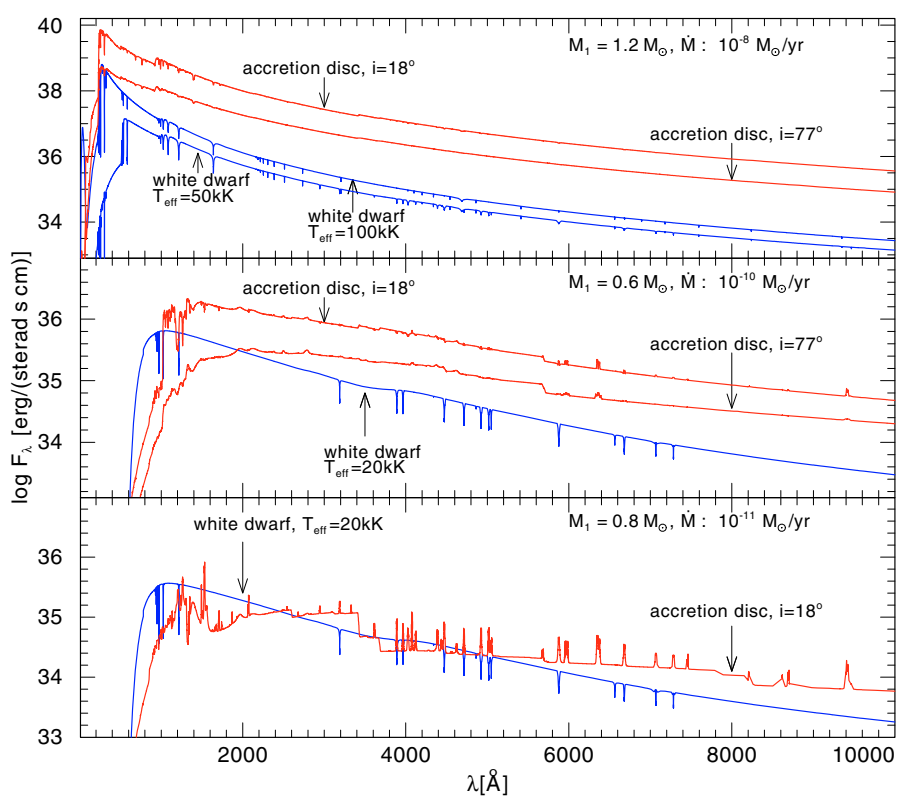

Fig. 14. Comparison of spectra of accretion disc and white dwarf. Top panel: $1.2 M_{\odot}$ primary with $T_{\text {eff }}=50000 \mathrm{~K}$ and $T_{\text {eff }}=100000 \mathrm{~K}$ and $\dot{M}=10^{-10} M_{\odot} / \mathrm{yr}$, accretion disc extended between 1.3 and $20 R_{1}$. Middle panel: $0.6 M_{\odot}$ primary with $T_{\text {eff }}=20000 \mathrm{~K}$ and $\dot{M}=$ $10^{-10} M_{\odot} / \mathrm{yr}$, accretion disc extended between 1.3 and $12.5 R_{1}$. Bottom panel: $0.8 M_{\odot}$ primary with $T_{\text {eff }}=20000 \mathrm{~K}$ and $\dot{M}=10^{-11} M_{\odot} / \mathrm{yr}$, accretion disc extended between 1.3 and $18 R_{1}$.

correspond to the light of the white dwarf, the accretion disc, and the donor star. Only for one of the AMCVn systems the primary seems to have been spectroscopically detected (Sion et al. 2006). To investigate the amount to which the white dwarf contributes to the total spectrum, we calculated synthetic helium-dominated $\left(\mathrm{H} / \mathrm{He}=10^{-5}\right)$ spectra using our stellar atmosphere code TMAP. We then weighted the spectra with the WD surface area, ignoring partial occlusion by the accretion disc, and compared them with the accretion-disc spectra (Fig. 14). For high mass-accretion rates $\left(\dot{M}=10^{-8} M_{\odot} / \mathrm{yr}\right)$, we assumed a hot WD of $50000 \mathrm{~K}$ and $100000 \mathrm{~K}$. We found that the flux

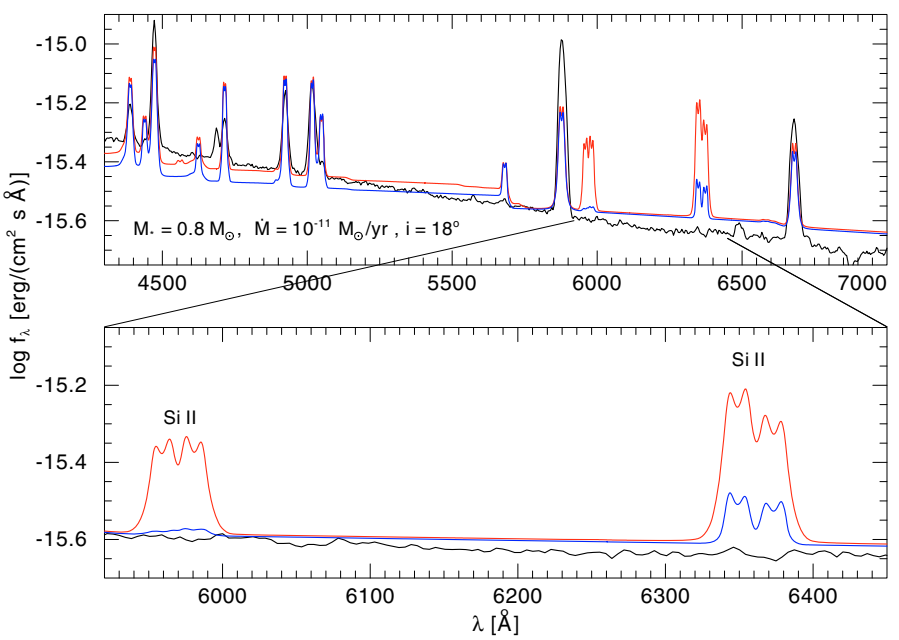

Fig. 15. Comparison of the spectrum of CE 315 (Ruiz et al. 2001, thick line) with a model spectrum with solar and 0.001 times solar silicon abundance (lower panel, zoom). The mass of the primary is $0.8 M_{\odot}$, the mass-accretion rate is $10^{-11} M_{\odot} / y r$, the inclination is $18^{\circ}$. The He I lines in the observed spectrum are dominated by a component probably not created in the disc itself but at the hotspot or the inner boundary layer (as discussed by Ruiz et al. 2001).

of even the coolest disc is higher than the flux of the related white dwarf $\left(T_{\text {eff }}=50000 \mathrm{~K}\right)$. At low mass-accretion rate $(\dot{M}=$ $\left.10^{-10} M_{\odot} / \mathrm{yr}\right)$, assuming a WD of $20000 \mathrm{~K}$, there is a chance of detecting the primary in the UV, especially if the disc is at high inclination. In the optical and infrared range, the disc flux is much higher than that of the white dwarf, even if we reduce the disc size drastically (3-7 $R_{1}$ instead of $1.3-12.5 R_{1}$ ). The optimal opportunity of detecting the WD spectroscopically is in systems with the lowest mass-accretion rate $\left(\dot{M}=10^{-11} M_{\odot} / y r\right.$, again assuming $T_{\text {eff }}=20000 \mathrm{~K}$ ).

\section{Model versus observation in the case of CE 315}

CE 315 is the AMCVn system with the longest known orbital period $(65.1 \mathrm{~min})$. The optical spectrum shows emission lines of He I and He II, and a few weak N I and N II lines. The accretor mass is about $0.77 M_{\odot}$ (Ruiz et al. 2001). The profile of the spectral lines is variable on a timescale of a few minutes, probably due to the rotating hotspot, the collisional region between accretion stream and accretion disc. The helium lines often show three peaks. Ruiz et al. (2001) suggested that the outer components originate in the accretion disc, whereas the central peak is produced by the boundary layer. In the case of He II $\lambda 4686 \AA$ the central component dominates the line completely. This central peak feature is also seen in GPCom (Smak 1975; Morales-Rueda et al. 2003) and is much weaker in SDSS J124058.03-015919.2 (Roelofs et al. 2005). Because of the absence of silicon lines in the UV (Gänsicke et al. 2003) and optical spectra, CE 315 is thought to be a Population II object (Roelofs et al. 2005; Ramsay et al. 2006). A strong silicon underabundance $\left(10^{-3}\right.$ times solar) has also been found for GP Com (Marsh et al. 1991). From the analysis of the X-ray spectrum of CE 315, Ramsay et al. (2006) found a significant enhancement of nitrogen (68-92\% more than the solar value).

In Fig. 15, we compare an observed spectrum of CE 315 (Ruiz et al. 2001) with two model spectra with solar and 


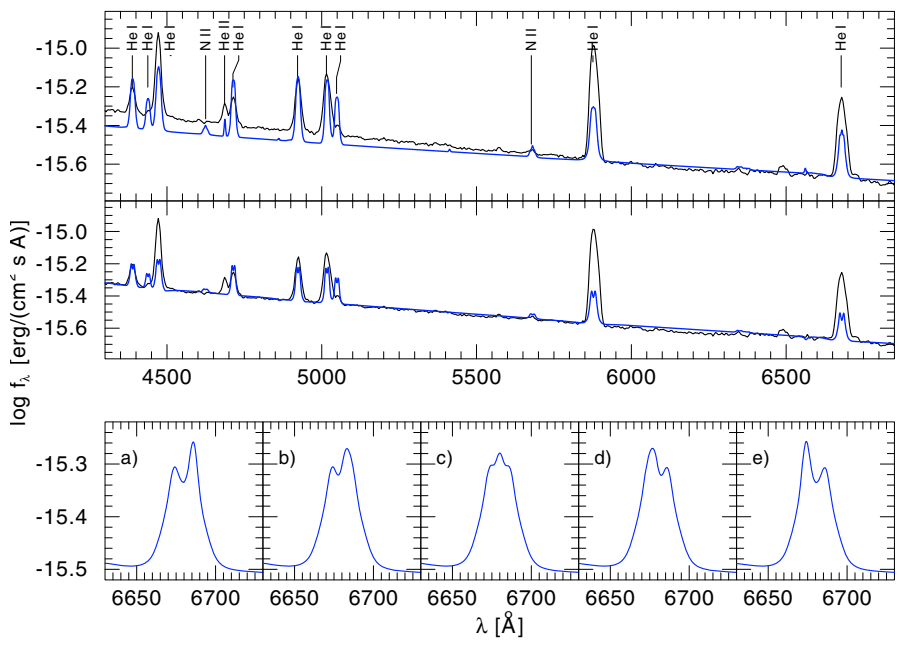

Fig. 16. Top panel: comparison of the spectrum of CE 315 (Ruiz et al. 2001, thick line) with a model with 0.0001 times solar silicon abundance, a boundary layer and a hotspot region. Middle: accretion disc without boundary layer and hotspot, combined with the model spectrum of a white dwarf $\left(T_{\text {eff }}=20000 \mathrm{~K}, \log g=8.3\right)$, compared with the observed spectrum. Lower panel: modulation of the He I $\lambda 6678 \AA$ spectral line caused by the orbiting hotspot. The position of the hotspot varies form a) moving away from observer to e) moving towards observer. Inclination of all models is $18^{\circ}$.

0.001 times solar silicon abundance. We assumed $M_{1}=0.8 M_{\odot}$ and $\dot{M}=10^{-11} M_{\odot} /$ yr. It is clearly indicative of a strong silicon underabundance $(<0.001$ times solar $)$ in the accretion disc of CE 315.

Since the model spectra are too flat, we added a hotter disc ring to the innermost part $\left(1.1-1.4 \mathrm{R}_{1}\right)$ of the disc to approximate a type of a boundary layer. Using a ring of $\dot{M}=10^{-9} M_{\odot} / \mathrm{yr}$, the flux in the UV becomes too high, in contrast to the observation showing almost no continuum in UV (Gänsicke et al. 2003). With a mass-accretion rate of $10^{-10} M_{\odot} / y r$, the flux in the UV is comparable to that of observations but in the optical still remains too flat. In addition to this boundary layer, we added to the outermost part of the disc a hot area approximating a hot-spot region in the following way. We took the structure of the outermost disc ring and heated the upper layers artificially from about $10000 \mathrm{~K}$ to $35000 \mathrm{~K}$. The result was an almost identical emission-line spectrum in the optical but of a higher flux level. In the final integration of all disc rings, this region was extended 20 degrees in azimuth and between 12 and $13.5 R_{1}$ in radial direction, representing the hot-spot region. Finally, we reduced the silicon abundance to 0.0001 times solar and set the nitrogen abundance to 2 times solar. The resulting model spectrum is shown in the upper panel of Fig. 16. In general, the observed spectrum is reproduced well, but some of the helium lines are too weak, and others are too strong.

We also determined the possible contribution of the white dwarf to the observed spectrum. In the middle panel of Fig. 16, the combined model spectrum of the pure disc (no boundary region or hotspot) and a white dwarf ( $T_{\text {eff }}=20000 \mathrm{~K}, \log g=8.3$ ) is compared with observations. The observed spectrum is reproduced qualitatively well indicating that the WD's contribution should not be neglected. Because of to the missing hotspot, the spectral lines are double peaked.

The lower panel of Fig. 16 shows in detail He I $\lambda 6678 \AA$. The influence of the orbiting hotspot on the line profile is obvious.

\section{Summary}

We have presented our grid of NLTE accretion-disc models for AM CVn systems. In the framework of the Virtual Observatory ${ }^{1}$ $(V O)$, all spectral energy distributions (SEDs, $\lambda-F_{\lambda}$ ) described here will be available in $V O$ compliant form from the VO service TheoSSA ${ }^{2}$ provided by the German Astrophysical Virtual Observatory $\left(G A V O^{3}\right)$. We used primary masses between $0.6 M_{\odot}$ and $1.4 M_{\odot}$ and mass-accretion rates between $10^{-11}$ and $10^{-8} M_{\odot} / \mathrm{yr}$ and varied the chemical composition. Our main results are as follows:

1. Discs with high mass-accretion rate $\left(10^{-8} M_{\odot} / \mathrm{yr}\right)$ show absorption-line spectra, and discs with low mass-accretion rate $\left(10^{-10}-10^{-11} M_{\odot} / \mathrm{yr}\right)$ emission-line spectra, as expected from observations.

2. Underabundances of $\mathrm{C}, \mathrm{O}$, and $\mathrm{Si}$ should be detectable by comparisons with observed spectra. A hydrogen abundance of $10^{-5}$ (mass fraction) leads to strong Balmer emission lines in low-state systems. In high-state systems, an abundance of $10^{-3}$ is necessary to obtain detectable Balmer lines. In both cases, it is nearly impossible to detect hydrogen lines in the UV.

3. Irradiation of the accretion disc by the primary ( $T_{\text {eff }}=20000 \mathrm{~K}$ for low-state and outbursting systems, $T_{\text {eff }}=50000 \mathrm{~K}$ up to $T_{\text {eff }}=100000 \mathrm{~K}$ for high-state systems) heats up the upper layers of the disc but has almost no effect on the spectrum.

4. Comparing the spectra of the primary white dwarf and the accretion disc shows that the primary, if at all, can be seen only in UV. The optical spectral range is dominated by the disc for $\dot{M}$ between $10^{-8}$ and $10^{-10} M_{\odot} /$ yr. Only for the lowest rate of $10^{-11} M_{\odot} / \mathrm{yr}$ is the continuum flux of the white dwarf in the optical higher than the disc flux.

5. The accretion-disc spectrum of CE 315 is reproduced well by assuming a $0.8 M_{\odot}$ primary, a mass-accretion rate of $10^{-11} M_{\odot} / \mathrm{yr}$, and a white dwarf with $T_{\text {eff }}=20000 \mathrm{~K}$, and $\log g=8.3$.

6. An orbiting hotspot in the outer part of the disc produces a modulation in the helium-line profiles.

7. We have confirmed a strong silicon underabundance in the accretion disc of CE 315 and, hence, that this system might be a Population II object.

Acknowledgements. We thank M. T. Ruiz for providing us with the observed spectrum of CE 315. T.R. is supported by the German Astrophysical Virtual Observatory (GAVO) project of the German Federal Ministry of Education and Research (BMBF) under grant 05 AC6VTB.

\section{References}

Anderson, S. F., Haggard, D., Homer, L., et al. 2005, AJ, 130, 2230

Anderson, S. F., Becker, A. C., Haggard, D., et al. 2008, AJ, 135, 2108

Bildsten, L., Townsley, D. M., Deloye, C. J., \& Nelemans, G. 2006, ApJ, 640, 466

El-Khoury, W., \& Wickramasinghe, D. 2000, A\&A, 358, 154

Gänsicke, B. T., Szkody, P., de Martino, D., et al. 2003, ApJ, 594, 443

Grevesse, N., \& Sauval, A. 2000, Solar Abundances, ed. P. Murdin, Encyclopedia of Astronomy and Astrophysics

Hils, D., \& Bender, P. L. 2000, ApJ, 537, 334

Kunze, S., Speith, R., \& Hessman, F. V. 2001, MNRAS, 322, 499

Marsh, T. R., Horne, K., \& Rosen, S. 1991, ApJ, 366, 535

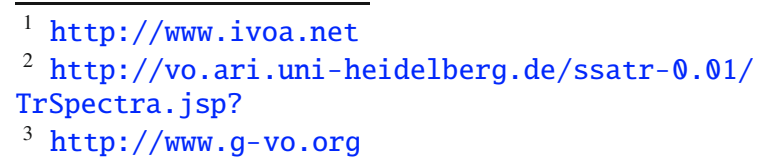


Morales-Rueda, L., Marsh, T. R., Steeghs, D., et al 2003, A\&A, 405, 249 Nagel, T., Dreizler, S., Rauch, T., \& Werner, K. 2004, A\&A, 428, 109 Nasser, M. R., Solheim, J.-E., \& Semionoff, D. A. 2001, A\&A, 373, 222

Nelemans, G. 2005, in The Astrophysics of Cataclysmic Variables and Related Objects, ed. J.-M. Hameury, \& J.-P. Lasota, ASP Conf. Ser., 330, 27

Nelemans, G., Portegies Zwart, S. F., Verbunt, F., \& Yungelson, L. R. 2001, A\&A, 368, 939

Podsiadlowski, P., Han, Z., \& Rappaport, S. 2003, MNRAS, 340, 1214

Ramsay, G., Groot, P. J., Marsh, T., et al. 2006, A\&A, 457, 623

Rauch, T., \& Deetjen, J. L. 2003, in Stellar Atmosphere Modeling, ed. I. Hubeny, D. Mihalas, \& K. Werner, ASP Conf. Ser., 288, 103

Roelofs, G. H. A., Groot, P. J., Marsh, T. R., et al. 2005, MNRAS, 361, 487

Roelofs, G. H. A., Groot, P. J., Nelemans, G., Marsh, T. R., \& Steeghs, D. 2007a, MNRAS, 379, 176
Roelofs, G. H. A., Groot, P. J., Steeghs, D., Marsh, T. R., \& Nelemans, G. 2007b, MNRAS, 382, 1643

Ruiz, M. T., Rojo, P. M., Garay, G., \& Maza, J. 2001, ApJ, 552, 679

Semionovas, D., \& Solheim, J.-E. 1999, in 11th European Workshop on White Dwarfs, ed. J.-E. Solheim, \& E. G. Meistas, ASP Conf. Ser., 169, 356

Shakura, N. I., \& Sunyaev, R. A. 1973, A\&A, 24, 337

Sion, E. M., Solheim, J.-E., Szkody, P., Gaensicke, B. T., \& Howell, S. B. 2006, ApJ, 636, L125

Smak, J. 1975, Acta Astron., 25, 371

Solheim, J.-E., \& Yungelson, L. R. 2005, in 14th European Workshop on White Dwarfs, ed. D. Koester, \& S. Moehler, ASP Conf. Ser., 334, 387

Tsugawa, M., \& Osaki, Y. 1997, PASJ, 49, 75

Warner, B. 1995, Cataclysmic variable stars, Cambridge Astrophysics Series (Cambridge, New York: Cambridge University Press) 